\title{
On the difference between a D. H. Lehmer number and its inverse modulo $q$
}

\author{
by
}

WenPENG ZHANG (Xi'an)

1. Introduction. Let $q>2$ be an odd integer. For each integer $x$ with $0<x<q$ and $(q, x)=1$, we know that there exists one and only one $\bar{x}$ with $0<\bar{x}<q$ such that $x \bar{x} \equiv 1(\bmod q)$. Let $r(q)$ be the number of cases in which $x$ and $\bar{x}$ are of opposite parity. For $q=p$ a prime, D. H. Lehmer [2] asks us to find $r(p)$ or at least to say something nontrivial about it. For the sake of simplicity, we call such a number $x$ as a $D$. H. Lehmer number. The main purpose of this paper is to study the distribution properties of D. H. Lehmer numbers and the asymptotic properties of the $2 k$ th power mean

$$
M(q, k)=\sum_{\substack{a=1 \\ 2 \mid a+\bar{a}+1}}^{q}(a-\bar{a})^{2 k}
$$

where $\sum_{a}^{\prime}$ denotes the summation over all $a$ such that $(a, q)=1$.

It seems that no one has studied this problem yet; at least I have not seen expressions like (1) before. The problem is interesting because it can help us to find how large is the difference between a D. H. Lehmer number and its inverse modulo $q$. In this paper, we use estimates of Kloosterman's sums and properties of trigonometric sums to give a sharper asymptotic formula for $M(q, k)$ for any fixed positive integer $k$. That is, we shall prove the following:

THEOREM 1. For any odd number $q$ and integer $k$, we have the asymptotic formula

$$
M(q, k)=\frac{1}{(2 k+1)(2 k+2)} \varphi(q) q^{2 k}+O\left(4^{k} q^{(4 k+1) / 2} \tau^{2}(q) \ln ^{2} q\right)
$$

where $\varphi(q)$ is the Euler function and $\tau(q)$ is the divisor function.

Project supported by the National Natural Science Foundation of China. 
For $0 \leq x, y \leq 1$, define the distribution function of $a, \bar{a}$ as

$$
F_{q}(x, y) \equiv \#\{a: a \leq x q, \bar{a} \leq y q, 2 \nmid a+\bar{a}\} .
$$

Then we can deduce the following limiting distribution theorem:

TheOREM 2. For any odd number $q>2$, we have

$$
F_{q}(x, y)=\frac{1}{2} x y \varphi(q)+O\left(q^{1 / 2} \tau^{2}(q) \ln ^{2} q\right) .
$$

From the theorems we can immediately deduce the following two corollaries:

COROllaRY 1. For any odd prime number $p$, we have the asymptotic formula

$$
M(p, 2)=\frac{1}{30} p^{5}+O\left(p^{9 / 2} \ln ^{2} p\right) .
$$

COROLlary 2. For any real numbers $0 \leq x, y \leq 1$, we have

$$
\lim _{q \rightarrow \infty} \frac{F_{q}(x, y)}{\varphi(q)}=\frac{1}{2} x y .
$$

2. Some elementary lemmas. In this section, we prove some elementary lemmas which are necessary in the proof of the theorems.

Lemma 1. Let $q$ be an odd number. For any integer $n$ and nonnegative integer $r$, define

$$
K(n, r)=\sum_{a=1}^{q} a^{r} e(a n / q), \quad H(n, r)=\sum_{a=1}^{q}(-1)^{a} a^{r} e(a n / q)
$$

where $e(y)=e^{2 \pi i y}$. We have the estimates

$$
\begin{gathered}
K(n, r) \begin{cases}=\frac{q^{r+1}}{r+1}+O\left(q^{r}\right) & \text { if } q \mid n, \\
\ll \frac{q^{r}}{|\sin (\pi n / q)|} \quad \text { if } q \nmid n,\end{cases} \\
H(n, r) \ll \frac{q^{r}}{|\cos (\pi n / q)|} .
\end{gathered}
$$

Proof. First we prove (2). For $r=0$, from the trigonometric identity

$$
\sum_{a=1}^{q} e\left(\frac{a n}{q}\right)= \begin{cases}n, & q \mid n, \\ 0, & q \nmid n,\end{cases}
$$

we immediately deduce that (2) holds. 
For $r>0$, if $q \nmid n$, then

$$
\begin{aligned}
K(n, r)\left(1-e\left(\frac{n}{q}\right)\right) & =\left(1-e\left(\frac{n}{q}\right)\right) \sum_{a=1}^{q} a^{r} e\left(\frac{a n}{q}\right) \\
& =\sum_{a=1}^{q} a^{r} e\left(\frac{a n}{q}\right)-\sum_{a=1}^{q} a^{r} e\left(\frac{(a+1) n}{q}\right) \\
& =\left(1-q^{r}\right) e\left(\frac{n}{q}\right)+\sum_{a=1}^{q-1}\left((a+1)^{r}-a^{r}\right) e\left(\frac{n(a+1)}{q}\right) \\
& \ll q^{r}+\sum_{a=1}^{q-1}\left((a+1)^{r}-a^{r}\right) \ll q^{r} .
\end{aligned}
$$

From this we can deduce that

$$
|K(n, r)| \ll \frac{q^{r}}{|\sin (\pi n / q)|} .
$$

If $q \mid n$, then from Euler's summation formula we get

$$
\begin{aligned}
K(n, r) & =\sum_{a=1}^{q} a^{r} e\left(\frac{a n}{q}\right)=\sum_{a=1}^{q} a^{r} \\
& =\int_{0}^{q} x^{r} d x+O\left(q^{r}\right)=\frac{q^{r+1}}{r+1}+O\left(q^{r}\right) .
\end{aligned}
$$

Combining (5) and (6) we obtain (2).

Now we prove (3). If $q \mid n$, then

$$
H(n, r)=\sum_{a=1}^{q}(-1)^{a} a^{r} e\left(\frac{a n}{q}\right)=\sum_{a=1}^{q}(-1)^{a} a^{r} \ll q^{r} .
$$

If $q \nmid n$, then

(8) $H(n, r)\left(1+e\left(\frac{n}{q}\right)\right)$

$$
\begin{aligned}
& =\left(1+e\left(\frac{n}{q}\right)\right) \sum_{a=1}^{q}(-1)^{a} a^{r} e\left(\frac{a n}{q}\right) \\
& =\sum_{a=1}^{q}(-1)^{a} a^{r} e\left(\frac{a n}{q}\right)+\sum_{a=1}^{q}(-1)^{a} a^{r} e\left(\frac{(a+1) n}{q}\right) \\
& \ll q^{r}+\sum_{a=1}^{q-1}\left((a+1)^{r}-a^{r}\right) \ll q^{r} .
\end{aligned}
$$


Noting that $|1+e(n / q)|=2|\cos (\pi n / q)|$, (3) follows from (7) and (8). This completes the proof of Lemma 1.

Lemma 2. Let $m, n$ and $q$ be integers, and $q>2$. Then we have the estimates

$$
S(m, n ; q)=\sum_{\substack{d(\bmod q) \\(d, q)=1}} e\left(m \frac{\bar{d}}{d}+n \frac{d}{q}\right) \ll(m, n, q)^{1 / 2} q^{1 / 2} \tau(q)
$$

where $d \bar{d} \equiv 1(\bmod q), \tau(q)$ is the divisor function, $(m, n, q)$ is the greatest common factor of $m, n$ and $q$, and $e(y)=e^{2 \pi i y}$.

Proof. See [1].

Lemma 3. Let $r, s$ and $q$ be positive integers and $q>2$. Then

$$
\sum_{\substack{a=1 \\ a b \equiv 1(q)}}^{q} \sum_{\substack{b=1 \\ \prime}}^{q} a^{r} b^{s}=\frac{\varphi(q) q^{r+s}}{(r+1)(s+1)}+O\left(q^{r+s+1 / 2} \tau^{2}(q) \ln ^{2} q\right)
$$

where $\varphi(q)$ is the Euler function.

Proof. First notice that from (4) we get the identity

(9)

$$
\begin{aligned}
& \sum_{\substack{a=1 \\
a b \equiv 1(q)}}^{q} \sum_{\substack{b=1 \\
q^{\prime}}}^{\prime} a^{r} b^{s} \\
& \quad=\frac{1}{q^{2}} \sum_{\substack{a=1 \\
a b \equiv 1(q)}}^{q} \sum_{\substack{b=1 \\
c, d=1}}^{q} \sum^{r} d^{s} \sum_{m, n=1}^{q} e\left(\frac{m(a-c)+n(b-d)}{q}\right) \\
& \quad=\frac{1}{q^{2}} \sum_{m, n=1}^{q}\left(\sum_{\substack{a=1 \\
a b \equiv 1}}^{q} \sum_{\substack{b=1 \\
(q)}}^{q} e\left(\frac{a m+b n}{q}\right)\right) \\
& \quad \equiv \frac{1}{q^{2}} \sum_{m=1}^{q} \sum_{n=1}^{q} S(m, n ; q) K(-m, r) K(-n, s) \\
& \quad=\frac{1}{q^{2}} \sum_{m=1}^{q-1} S(m, q ; q) K(-m, r) K(-q, s)
\end{aligned}
$$




$$
\begin{aligned}
& +\frac{1}{q^{2}} \sum_{n=1}^{q-1} S(q, n ; q) K(-q, r) K(-n, s) \\
& +\frac{1}{q^{2}} \sum_{m=1}^{q-1} \sum_{n=1}^{q-1} S(m, n ; q) K(-m, r) K(-n, s) \\
& +\frac{1}{q^{2}} S(q, q ; q) K(-q, r) K(-q, s)
\end{aligned}
$$

where $K(-m, r)$ is defined in Lemma 1. From (2) of Lemma 1, Lemma 2 and noting that $2 / \pi \leq(\sin x) / x$ for $|x| \leq \pi / 2$, we get

(10) $\frac{1}{q^{2}} S(q, q ; q) K(-q, r) K(-q, s)$

$$
\begin{aligned}
& =\frac{\varphi(q)}{q^{2}}\left(\frac{q^{r+1}}{r+1}+O\left(q^{r}\right)\right)\left(\frac{q^{s+1}}{s+1}+O\left(q^{s}\right)\right) \\
& =\frac{\varphi(q) q^{r+s}}{(r+1)(s+1)}+O\left(q^{r+s}\right),
\end{aligned}
$$

(11) $\sum_{m=1}^{q-1} S(m, q ; q) K(-m, r) K(-q, s)$

$$
\begin{aligned}
& \ll \sum_{m=1}^{q-1} q^{1 / 2}(m, q)^{1 / 2} \tau(q) q^{s+1} \frac{q^{r}}{|\sin (\pi m / q)|} \\
& \ll q^{r+s+5 / 2} \tau(q) \sum_{m=1}^{q-1} \frac{(m, q)^{1 / 2}}{m} \ll q^{r+s+5 / 2} \tau^{2}(q) \ln q .
\end{aligned}
$$

Similarly, we can get the estimates

$$
\begin{aligned}
& \sum_{n=1}^{q-1} S(q, n ; q) K(-q, r) K(-n, s) \ll q^{r+s+5 / 2} \tau^{2}(q) \ln q, \\
& \text { (13) } \sum_{m=1}^{q-1} \sum_{n=1}^{q-1} S(m, n ; q) K(-m, r) K(-n, s) \\
& \ll \sum_{m=1}^{q-1} \sum_{n=1}^{q-1} \frac{q^{1 / 2} \tau(q)(m, n, q)^{1 / 2} q^{r+s}}{|\sin (\pi m / q)||\sin (\pi n / q)|} \\
& \ll q^{r+s+5 / 2} \tau(q) \sum_{m=1}^{q-1} \sum_{n=1}^{q-1} \frac{(m, n, q)^{1 / 2}}{m n} \\
& \ll q^{r+s+5 / 2} \tau(q) \ln ^{2} q .
\end{aligned}
$$


Combining (9)-(13) we immediately deduce that

$$
\sum_{\substack{a=1 \\ a b \equiv 1(q)}}^{q} \sum_{\substack{b=1 \\ \prime}}^{q} a^{r} b^{s}=\frac{\varphi(q) q^{r+s}}{(r+1)(s+1)}+O\left(q^{r+s+1 / 2} \tau^{2}(q) \ln ^{2} q\right) .
$$

This is the conclusion of Lemma 3 .

Lemma 4. Let $r, s$ and $q$ be positive integers and $q>2$. Then

$$
\sum_{\substack{a=1 \\ a b \equiv 1(q)}}^{q} \sum_{\substack{b=1 \\ q}}^{q}(-1)^{a+b} a^{r} b^{s}=O\left(q^{r+s+1 / 2} \tau^{2}(q) \ln ^{2} q\right) .
$$

Pr o of. Similarly, we get

$$
\begin{aligned}
& \sum_{\substack{a=1 \\
a b \equiv 1(q)}}^{q} \sum_{\substack{b=1 \\
\text { q }}}^{q}(-1)^{a+b} a^{r} b^{s} \\
& =\frac{1}{q^{2}} \sum_{a=1}^{q} \sum_{b=1}^{q} \sum_{c, d=1}^{q}(-1)^{c+d} c^{r} d^{s} \sum_{m, n=1}^{q} e\left(\frac{m(a-c)+n(b-d)}{q}\right) \\
& a b \equiv 1(q) \\
& =\frac{1}{q^{2}} \sum_{m, n=1}^{q}\left(\sum_{\substack{a=1 \\
a b \equiv 1(q)}}^{q} \sum_{\substack{b=1 \\
\prime}}^{q} e\left(\frac{m a+n b}{q}\right)\right) \\
& \times\left(\sum_{c, d=1}^{q}(-1)^{c+d} c^{r} d^{s} e\left(\frac{-m c-n d}{q}\right)\right) \\
& \equiv \frac{1}{q^{2}} \sum_{m=1}^{q} \sum_{n=1}^{q} S(m, n ; q) H(-m, r) H(-n, s)
\end{aligned}
$$

where $H(-m, r)$ is defined in Lemma 1.

Noting that $|\cos (\pi m / q)|=|\sin (\pi(q-2 m) /(2 q))|$ and $q-2 m \neq 0$, from (3) of Lemma 1, (14) and using the method of proof of Lemma 3 we easily deduce the conclusion of Lemma 4.

Lemma 5. Let $q>2$ be an odd number. Then for any fixed $0 \leq x, y \leq 1$ we have

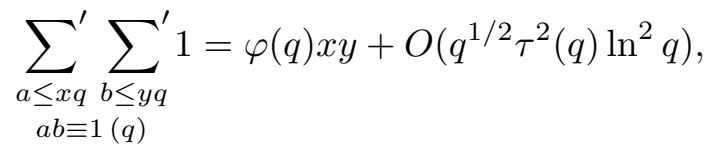




$$
\sum_{\substack{a \leq x q \\ a b \equiv 1(q)}}^{\prime} \sum_{\substack{b \leq y q \\(-1)^{a+b}}}^{\prime}=O\left(q^{1 / 2} \tau^{2}(q) \ln ^{2} q\right) .
$$

Pro of. For fixed $0 \leq x, y \leq 1$, we define

$$
K(x, n, q)=\sum_{a \leq x q} e\left(\frac{a n}{q}\right), \quad H(x, n, q)=\sum_{a \leq x q}(-1)^{a} e\left(\frac{a n}{q}\right) .
$$

Using the method of proving Lemma 1 we can get the estimates

$$
\begin{gathered}
K(x, n, q) \begin{cases}=x q+O(1) & \text { if } q \mid n, \\
\ll \frac{1}{|\sin (\pi n / q)|} & \text { if } q \nmid n,\end{cases} \\
H(x, n, q) \ll \frac{1}{|\cos (\pi n / q)|} .
\end{gathered}
$$

From (15), Lemma 2 and the method of proof of Lemma 3 we easily deduce that

$$
\begin{aligned}
\sum_{\substack{a \leq x q \\
a b \equiv 1(q)}}^{\prime} \sum_{\substack{b \leq y q \\
q^{2}}}^{\prime} & =\frac{1}{q^{2}} \sum_{m=1}^{q} \sum_{n=1}^{q} S(m, n ; q) K(x,-m, q) K(y,-n, q) \\
& =\varphi(q) x y+O\left(q^{1 / 2} \tau^{2}(q) \ln ^{2} q\right) .
\end{aligned}
$$

This completes the proof of (I).

Similarly, from (16), Lemma 2 and the method of proof of Lemma 4 we deduce (II).

3. Proof of the theorems. First we prove Theorem 1. By the binomial formula, Lemma 3 and Lemma 4 we get

$$
\begin{aligned}
M(q, k) & =\sum_{\substack{a=1 \\
2 \mid a+\bar{a}+1}}^{q}(a-\bar{a})^{2 k}=\frac{1}{2} \sum_{\substack{a=1 \\
a b \equiv 1(q)}}^{q} \sum_{\substack{b=1 \\
q}}^{\prime}\left(1-(-1)^{a+b}\right)(a-b)^{2 k} \\
& =\frac{1}{2} \sum_{a=1}^{q}(a-\bar{a})^{2 k}-\frac{1}{2} \sum_{a=1}^{q}(-1)^{a+\bar{a}}(a-\bar{a})^{2 k} \\
& =\frac{1}{2} \sum_{i=0}^{2 k}\left(\begin{array}{c}
2 k \\
i
\end{array}\right)(-1)^{i}\left(\sum_{a=1}^{q} a^{2 k-i}(\bar{a})^{i}-\sum_{a=1}^{q}(-1)^{a+\bar{a}} a^{2 k-i}(\bar{a})^{i}\right)
\end{aligned}
$$




$$
\begin{aligned}
& =\frac{1}{2} \sum_{i=0}^{2 k}\left(\begin{array}{c}
2 k \\
i
\end{array}\right)(-1)^{i}\left(\frac{\varphi(q) q^{2 k}}{(i+1)(2 k-i+1)}\right. \\
& \left.+O\left(q^{2 k+1 / 2} \tau^{2}(q) \ln ^{2} q\right)\right) \\
& +O\left(\sum_{i=0}^{2 k}\left(\begin{array}{c}
2 k \\
i
\end{array}\right) q^{2 k+1 / 2} \tau^{2}(q) \ln ^{2} q\right) \\
& =\frac{\varphi(q) q^{2 k}}{2} \sum_{i=0}^{2 k} \frac{(-1)^{i}\left(\begin{array}{c}
2 k \\
i
\end{array}\right)}{(i+1)(2 k-i+1)}+O\left(4^{k} q^{2 k+1 / 2} \tau^{2}(q) \ln ^{2} q\right) \\
& =\frac{\varphi(q) q^{2 k}}{2(2 k+1)(2 k+2)} \sum_{i=0}^{2 k}(-1)^{i}\left(\begin{array}{c}
2 k+2 \\
i+1
\end{array}\right) \\
& +O\left(4^{k} q^{2 k+1 / 2} \tau^{2}(q) \ln ^{2} q\right) \\
& =\frac{\varphi(q) q^{2 k}}{2(2 k+1)(2 k+2)}\left(-\sum_{i=0}^{2 k+2}(-1)^{i}\left(\begin{array}{c}
2 k+2 \\
i
\end{array}\right)+2\right) \\
& +O\left(4^{k} q^{2 k+1 / 2} \tau^{2}(q) \ln ^{2} q\right) \\
& =\frac{\varphi(q) q^{2 k}}{2(2 k+1)(2 k+2)}\left(-(1-1)^{2 k+2}+2\right)+O\left(4^{k} q^{2 k+1 / 2} \tau^{2}(q) \ln ^{2} q\right) \\
& =\frac{\varphi(q) q^{2 k}}{(2 k+1)(2 k+2)}+O\left(4^{k} q^{2 k+1 / 2} \tau^{2}(q) \ln ^{2} q\right) \text {. }
\end{aligned}
$$

This is the conclusion of Theorem 1.

Applying Lemma 5 we can deduce that

$$
\begin{aligned}
F_{q}(x, y) & \equiv \#\{a: a \leq x q, \bar{a} \leq y q, 2 \nmid a+\bar{a}\} \\
& =\frac{1}{2} \sum_{\substack{a \leq x q \\
a b \equiv 1(q)}}^{\prime} \sum_{b \leq y q}^{\prime}\left(1-(-1)^{a+b}\right) \\
& =\frac{1}{2} \sum_{\substack{a \leq x q \\
a b \equiv 1(q)}}^{\prime} \sum_{b \leq y q}^{\prime} 1-\frac{1}{2} \sum_{\substack{a \leq x q \\
a b \equiv 1(q)}}^{\prime} \sum_{b \leq y q}^{\prime}(-1)^{a+b} \\
& =\frac{1}{2} \varphi(q) x y+O\left(q^{1 / 2} \tau^{2}(q) \ln ^{2} q\right) .
\end{aligned}
$$

This completes the proof of Theorem 2 .

Acknowledgements. The author expresses his gratitude to the referee for helpful comments. 


\section{References}

[1] T. Estermann, On Kloosterman's sums, Mathematika 8 (1961), 83-86.

[2] R. K. Guy, Unsolved Problems in Number Theory, Springer, 1981, 139-140.

[3] W. Zhang, A problem of D. H. Lehmer and its generalization (II), Compositio Math. 91 (1994), 47-56.

DEPARTMENT OF MATHEMATICS

NORTHWEST UNIVERSITY

XI'AN SHAANXI

PEOPLE'S REPUBLIC OF CHINA

Received on 2.11.1993

and in revised form on 21.5.1994 